\title{
A matrix-perturbation-theory-based optimal strategy for small-signal stability analysis of large-scale power grid
}

\author{
Yude Yang ${ }^{1 *} \mathbb{D}$, Jixing Zhao ${ }^{1}$, Hui Liu' ${ }^{1}$ Zhijun Qin ${ }^{1}$, Jun Deng ${ }^{2}$ and Junjian Qi ${ }^{3}$
}

\begin{abstract}
In this paper, a sensitivity matrix based approach is proposed to improve the minimum damping ratio. The proposed method also avoids burdensome deviation calculations of damping ratio of large-scale power grids when compared to the Small-Signal-Stability Constrained Optimal Power Flow (SSSC-OPF) approach. This is achieved using the Matrix Perturbation Theory (MPT) to deal with the 2 nd order sensitivity matrices, and the establishment of an optimal corrective control model to regulate the output power of generating units to improve the minimum damping ratio of power grids. Finally, simulation results on the IEEE 9-bus, IEEE 39-bus and a China 634-bus systems show that the proposed approach can significantly reduce the burden of deviation calculation, while enhancing power system stability and ensuring calculation accuracy.
\end{abstract}

Keywords: Matrix perturbation theory, 2nd order sensitivity, Optimal strategy, Small-signal stability

\section{Introduction}

Small-signal stability of power systems is critical to the system security of power grids due to large-scale connections of power grids [1,2], rapid development of Ultra High Voltage Alternating Current (UHVAC) transmission [3-5], high penetration of renewable energy sources, etc [6-9].

Many reported work have shown that damping controllers can enhance small-signal stability [10]. In practice, the re-dispatch of generator powers can also provide additional measure to ensure the small-signal stability of a power grid. Therefore, Small-Signal-Stability Constrained Optimal Power Flow (SSSC-OPF) has become a 'hot' research topic. This is because SSSC-OPF can achieve an appropriate security level while considering economic objectives and technical constraints [11].

SSSC-OPF has been reported for improvement of small-signal stability, but the existing work with high calculation precision is time-consuming due to large computation requirement when calculating the eigenvalue [10-15]. Eigenvalue sensitivity based Interior Point Methods (IPM) have been proposed to improve

\footnotetext{
* Correspondence: yangyude@gxu.edu.cn

${ }^{1}$ College of Electrical Engineering, Guangxi University, Nanning 530004, China Full list of author information is available at the end of the article
}

the power transfer capability with small-signal stability constraints $[10,11]$, which is an SSSC-OPF based Numerical Eigenvalue Sensitivity (NES-SSSC-OPF). However, these methods have to deal with the burden of heavy computation from the repetitive calculation of eigenvalues, and at the same time, high precision cannot be guaranteed due to the neglected high-order terms of the small-signal stability constraints. In [12], this expected-security-cost optimal power flow with small-signal stability constraints is addressed by the closed-form formula with extra computational burden to guarantee the calculation accuracy. Approximate-singular-value-sensitivity-based IPM is used to coordinate oscillation control in electricity market [13]. For the two methods proposed in $[12,13]$, both are very time-consuming for calculating the Hessian of small-signal stability constraints. An optimization method based on the sequential quadratic programming algorithm with Gradient Sampling [14] can ensure the global and efficient convergence of SSSC-OPF [15]. However, the computation with high precision is again time-consuming due to the complex in formula derivation and sampling process.

Matrix Perturbation Theory plays an important role in describing the changes in eigensolution [16-18] and has been applied for structural dynamics [19], automation control [20], design of power parameters [21], error 
analysis of power systems [22, 23], etc. In a power system, the sensitivity obtained by MPT can evaluate the key parameters to small-signal stability and can also be applied for power dispatch and parameter design [24]. In the case of small fluctuations in the operating parameters, the 1st-order sensitivity of MPT can obtain the results with sufficient precision [25]. Power systems show nonlinear characteristics, while larger disturbance causes abrupt change of power system parameters [26]. The 2nd-order control strategy tolerates a larger disturbance which can be regarded as a parameter disturbance of more than $20 \%$ variation [24]. Therefore, the 2 nd-order sensitivity of system parameters with high precision becomes a concern.

In this paper, Matrix Perturbation Theory based Optimal Strategy (MPT-OS), a hybrid method combining IPM and the 2nd-order MPT is proposed to deal with the sensitivity of damping ratio for improving of the small-signal stability. The optimal calibration model is established to improve system stability by IPM and the MPT is used to deal with the Hessian Matrix of the damping ratio. Since the Hessian Matrix calculation only needs the original state matrix and perturbation variables in MPT, deviation calculation of the 2nd-order sensitivities is not needed.

The remainder of this paper is organized as follows: In Section 2, the control model of small-signal stability and the partial derivative of damping ratio in SSSC-OPF are addressed. A MPT based computing method for the sensitivity of damping ratio is introduced in Section 3. In Section 4, the proposed approach is validated on three systems, and Section 5 draws the conclusion.

\section{Optimal model of small-signal stability}

\subsection{The optimal model}

The corrective control model can be described as follows:

1) The objective function.

The minimum change of the output powers of the generators is considered as the objective function as

$$
f(x)=\min \sum_{i \in S_{G}} \Delta P_{G i}^{2}
$$

where $i \in S_{G i}$ and $S_{G i}$ is the set of generators. $P_{G i}$ is the active power output of the generator and $P_{G i}^{0}$ is its initial active power output of the generator. $\Delta P_{G i}$ is the regulating output power of the generator given as $\Delta P_{G i}$ $=P_{G i}-P_{G i}^{0}$

2) The equality constraints.
The power balance equation is:

$$
\left\{\begin{array}{l}
P_{G i}-P_{D i}-V_{i} \sum_{j=1}^{n} Y_{i j} V_{j} \cos \left(\theta_{i}-\theta_{j}-\alpha\right)=0 \\
Q_{R i}-Q_{D i}-V_{i} \sum_{j=1}^{n} Y_{i j} V_{j} \sin \left(\theta_{i}-\theta_{j}-\alpha\right)=0
\end{array}\right.
$$

where $i \in S_{n}$ and $S_{n}$ is the set of nodes. $P_{D i}$ is the active load, $Q_{R i}$ is the reactive power output of the generator and $Q_{D i}$ is the reactive load; $K_{\lambda i}$ and $\theta_{i}$ are the voltage amplitude and phase angles respectively. $Y_{i j}$ and $\alpha_{i j}$ are the amplitude and phase angle of the admittance matrix, respectively.

3) Inequality constraints.

(1) Operation constraints are:

$$
\left\{\begin{array}{lc}
P_{G i}^{\min } \leq P_{G i} \leq P_{G i}^{\max } & i \in S_{G} \\
Q_{R i}^{\min } \leq Q_{R i} \leq Q_{R i}^{\max } & i \in S_{R} \\
V_{i}^{\min } \leq V_{i} \leq V_{i}^{\max } & i \in S_{n}
\end{array}\right.
$$

where $S_{R}$ is the set of reactive power supplies. $P_{G i}^{\min }$ and $P_{G i}^{\max }$ are the upper and lower limits of the active power output of the generator, respectively. $Q_{R i}^{\min }$ and $Q_{R i}^{\max }$ are the respective upper and lower limits of the reactive power output of the generator, $V_{i}^{\min }$ and $V_{i}^{\max }$ are the upper and lower limits of the voltage, respectively.

\section{(2) Stability constraint is:}

$$
\xi \geq \underline{\xi}
$$

where $\xi$ is the system damping ratio and $\xi$ is its lower limit. The index $\xi$ is to guarantee the system to be small-signal stable, and for $\xi<\xi$, the system has smallsignal stability problem.

After the improvement of system stability, $\xi=\xi_{0}+\Delta \xi$ where $\xi_{0}$ is the initial damping ratio, and $\Delta \xi$ can be written as:

$$
\Delta \xi=\sum_{i \in S_{G}}\left(K_{\xi} \Delta P_{G i}+\frac{1}{2} \Delta P_{G i} H_{\xi} \Delta P_{G i}\right)
$$

where $K_{\xi}$ and $H_{\xi}$ are the respective gradient matrix and Hessian matrix of the damping ratio and active power of the generators.

The small-signal stability corrective control model of (1) $-(5)$ can be described as:

$$
\min f\left(x_{0}\right)
$$

s.t. $h\left(x_{0}\right)=0$ 


$$
\underline{g} \leq g\left(x_{0}\right) \leq \bar{g}
$$

where $x_{0}$ is the initial state variable, $f(x)$ is the objective function, $h(x)$ and $g(x)$ are the equality and inequality constraints, respectively. $\bar{g}$ and $g$ are the upper and lower limits, respectively.

According to IPM, (8) can be written as $g\left(x_{0}\right)-l_{0}-\underline{g}=0$ and $g\left(x_{0}\right)+l_{0}-\bar{g}=0$, where $l_{0}$ is the slack variables with $l_{0}>0$.

Equations (6)-(8) can be written as Lagrange function as:

$$
L=f\left(x_{0}\right)-y^{T} h\left(x_{0}\right)-z^{T}\left(g\left(x_{0}\right)-l\right)-\mu \sum \ln (l)
$$

First-order optimality conditions (Karush Kuhn Tucker, KKT) are:

$$
\left\{\begin{array}{l}
L_{x}=\nabla_{x} f\left(x_{0}\right)-\nabla_{x} h\left(x_{0}\right) y-\nabla_{x} g\left(x_{0}\right) z=0 \\
L_{y}=\nabla h\left(x_{0}\right)=0 \\
L_{Z}=g\left(x_{0}\right)-l-g=0 \\
L_{l}=L Z e-\mu e=0
\end{array}\right.
$$

where $y$ and $z$ are the respective Lagrange multipliers, $\mu$ is the blockage parameter, $L$ and $Z$ are the diagonal matrix of $l$ and $z$, respectively, and $e$ is the unit column vector. The damping ratio constraints part of $\nabla_{x} g(x)$ can be written as $K_{\xi}$.

Using Newton's method to solve (10), the correction equation is obtained:

$$
\left[\begin{array}{cc}
H & \nabla_{x} h\left(x_{0}\right) \\
\nabla_{x}^{T} h\left(x_{0}\right) & 0
\end{array}\right]\left[\begin{array}{l}
\Delta x \\
\Delta y
\end{array}\right]=\left[\begin{array}{l}
L_{x}^{\prime} \\
L_{y}
\end{array}\right]
$$

where $H=\nabla_{x}^{2} f-\nabla_{x}^{2} h y-\nabla_{x}^{2} g z-\nabla_{x} g L^{-1} Z \nabla_{x}^{T} g$, and corrections are solved by iteration in (11). The damping ratio constraints part of $\nabla_{x}^{2} g(x)$ can be represent by $H_{\xi}$. Section 3 describes the detailed derivation process of $K_{\xi}$ and $H_{\xi}$.

The eigenvalue constraint has been considered in many studies, but the damping ratio constraint has been ignored. In this paper, the models of (1)-(5) are established in a similar way by IPM. However, derivative calculations of damping ratio are complex due to the implicit relationship between the generator active power and the damping ratio, which seriously affects the practicability of SSSC-OPF. The existing method [12] spends significant computation time on heavy eigenvalue computation of the QR method, which avoids derivative calculations. Therefore, it is necessary to effectively simplify computation in practical application of SSSC-OPF. Compared to the existing methods [12], the $K_{\xi}$ and $H_{\xi}$ are calculated by MPT in the presented method, which avoids demanding derivative calculations and reduces computing time.

\subsection{Corrective control process}

In Fig. 1, the steps of the small-signal stability optimal strategy are as follows.

1) Small signal stability analysis.

The system stability is judged by small-signal stability analysis.

2) Damping ratio sensitivity.

The eigenvalue and eigenvector sensitivities are calculated by MPT. The damping ratio sensitivity is deduced by eigenvalue and eigenvector sensitivities.

3) The corrective control model of power dispatch. The corrective control model is established to improve the system stability by regulating the output power of generating units.

4) Judgement of system stability.

The corrective control results are checked by small signal stability analysis. If the results meet the system stability requirement, the calculation is stopped, otherwise, the corrective control model is re-established to improve the system stability.

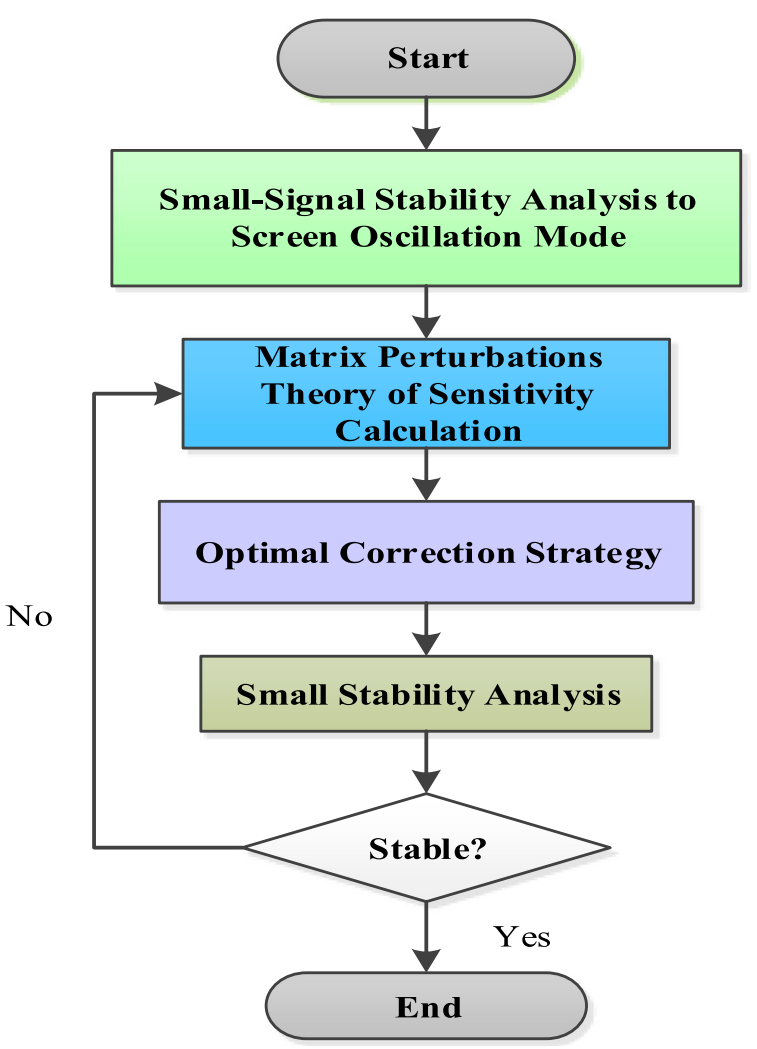

Fig. 1 Optimization correction procedures. The Figure show that the corrective control procedures of Matrix-PerturbationTheory-based Optimal Strategy to improve small-signal-stability 


\section{Methods: damping sensitivity matrixes}

\subsection{Matrix perturbation theory}

The calculation of $K_{\xi}$ and $H_{\xi}$ involves the change of state matrix, perturbation variable and initial eigensolution in MPT.

(1) Initial eigensolution

The state matrix is described as follows [27, 28]:

$$
\begin{aligned}
& A_{0}=\tilde{A}-\tilde{B} \tilde{D}^{-1} \tilde{C} \\
& \frac{d \Delta x}{d t}=A_{0} \Delta x
\end{aligned}
$$

where $\tilde{A}$ and $\tilde{B}$ are the coefficient matrixes of the state variables, $\tilde{C}$ and $\tilde{D}$ are the coefficient matrixes of the non-state variables, $A_{0}$ is the initial system state matrix, and $\Delta x$ is the small change of the system state variables. The initial eigensolution contains the initial matrix eigenvalue $\lambda_{0}$, initial left eigenvector $u_{0}$, initial right eigenvector $v_{0}$ and initial damping ratio $\xi_{0}$ :

$$
\begin{aligned}
& \left.\begin{array}{rl}
A_{0} v_{0} & =\lambda_{0} v_{0} \\
u_{0}{ }^{T} A_{0} & =\lambda_{0} u_{0}{ }^{T}
\end{array}\right\} \\
& \left\{\begin{array}{l}
\lambda_{0}=\alpha_{0}+j \beta_{0} \\
\xi_{0}=\frac{-\alpha_{0}}{\sqrt{\alpha_{0}^{2}+\beta_{0}^{2}}}
\end{array}\right.
\end{aligned}
$$

(2) Perturbation variable and the change of state matrix

When the perturbation variable is the output power of generating units $\Delta P_{G i}$, the change of state matrix $\Delta A$ can be expressed as:

$$
A=A_{0}+\Delta A
$$

where $A_{0}$ and $A$ are the original and new state matrixes, respectively.

The change of state matrix $\Delta A$ is written as:

$$
\begin{gathered}
\Delta A=\sum_{i \in S_{G}} A_{s i} \Delta P_{G i} \\
\left\{\begin{array}{c}
\Delta P_{G}=\left[\Delta P_{G 1}, \Delta P_{G 2}, \ldots, \Delta P_{G i}, \ldots .,\right]^{T} \\
A_{s}=\left[A_{s 1}, A_{s 2}, \ldots, A_{s i}, \ldots,\right]^{T}
\end{array}\right.
\end{gathered}
$$

where $A_{s i}$ is the sensitivity of the state matrix and is given as $A_{s i}=\Delta A_{i} / \Delta P_{G i}$.

(3) Eigenvalue sensitivity

The eigensolution of the uncertain relation problem is described as [21, 22]:

$$
\left(A_{0}+\Delta A\right)\left(v_{i 0}+\Delta v_{i}\right)=\left(\lambda_{i 0}+\Delta \lambda_{i}\right)\left(v_{i 0}+\Delta v_{i}\right) \quad i \in S_{L}
$$

where $S_{L}$ is the set of system state variables, $\lambda_{i 0}$ is the $i$ th initial eigenvalue, $v_{i 0}$ is the $i$ th initial right eigenvector, $\Delta \nu_{i}$ is the change of the $i$ th right eigenvector, and $\Delta \lambda_{i}$ is the change of the $i$ th eigenvalue. According to MPT, the eigenvalue $\lambda_{i}$ and eigenvector $v_{i}$ of (19) are represented by [16]:

$$
\left\{\begin{array}{l}
\lambda_{i}=\lambda_{i 0}+\lambda_{i 1}+\lambda_{i 2} \\
v_{i}=v_{i 0}+v_{i 1}+v_{i 2}
\end{array}\right.
$$

where $i \in S_{L}, \lambda_{i 1}$ and $v_{i 1}$ are the 1st-order perturbation values, and $\lambda_{i 2}$ and $v_{i 2}$ are the 2nd-order perturbation values.

Substituting (19) into (20) and ignoring the perturbation values equal or higher than the 3rd-order yield:

$$
\left\{\begin{array}{l}
o^{0}: A_{0} v_{i 0}=\lambda_{i 0} v_{i 0} \\
o^{1}: A_{0} v_{i 1}+\Delta A v_{i 0}=\lambda_{i 0} v_{i 1}+\lambda_{i 1} v_{i 1} \\
o^{2}: A_{0} v_{i 2}+\Delta A v_{i 1}=\lambda_{i 0} v_{i 2}+\lambda_{i 1} v_{i 1}+\lambda_{i 2} v_{i 0}
\end{array}\right.
$$

The 2nd-order Taylor expansion of (21) is described as

$$
\left\{\begin{array}{l}
\lambda_{i}=\lambda_{i 0}\left(P_{G}^{0}\right)+K_{\lambda i} \Delta P_{G}+\frac{1}{2} \Delta P_{G}{ }^{T} H_{\lambda i} \Delta P_{G} \\
v_{i}=v_{i 0}\left(P_{G}^{0}\right)+K_{v i} \Delta P_{G}+\frac{1}{2} \Delta P_{G}^{T} H_{v i} \Delta P_{G}
\end{array}\right.
$$

where $i \in S_{L}, K_{\lambda i}$ and $K_{u i}$ are the gradient matrixes of eigenvalue and eigenvector, respectively, and $H_{\lambda i}$ is the Hessian matrix of eigenvalue. The gradient matrix and Hessian matrix can be written as:

$$
\left\{\begin{array}{l}
K_{\lambda_{i}}=\left[\frac{\partial \Delta \lambda_{i}}{\partial \Delta P_{G 1}}, \frac{\partial \Delta \lambda_{i}}{\partial \Delta P_{G 2}}, \ldots, \frac{\partial \Delta \lambda_{i}}{\partial \Delta P_{G j}}, \ldots\right] \\
K_{v_{i}}=\left[\frac{\partial \Delta v_{i}}{\partial \Delta P_{G 1}}, \frac{\partial \Delta v_{i}}{\partial \Delta P_{G 2}}, \ldots, \frac{\partial \Delta v_{i}}{\partial \Delta P_{G j}}, \ldots\right]
\end{array}\right.
$$

$$
H_{\lambda_{i}}=\left[\begin{array}{ccc}
\frac{\partial^{2} \Delta \lambda_{i}}{\partial \Delta P_{G 1}^{2}} & \cdots & \frac{\partial^{2} \Delta \lambda_{i}}{\partial \Delta P_{G j} \partial \Delta P_{G 1}} \\
\frac{\partial^{2} \ddot{\Delta}_{i}}{\partial \Delta P_{G 1} \partial \Delta P_{G j}} & \cdots & \frac{\partial^{2} \ddot{\Delta}_{i}}{\partial \Delta P_{G j}^{2}}
\end{array}\right]
$$

where $i \in S_{L}$, and $j \in S_{G}$.

According to MPT and the normalized eigenvectors, the eigenfunctions are substituted into (15) and combined with (21) and (23). The 1st-order perturbations can be described as [22]:

$$
\lambda_{i 1}=u_{i 0}^{T} \Delta A v_{i 0}
$$




$$
v_{i 1}=\frac{\sum_{j=1}\left(-u_{j 0}^{T} \Delta A^{T} v_{i 0}+\lambda_{i 0} u_{j 0}^{T} v_{i 0}\right) v_{j 0}}{\lambda_{j 0}-\lambda_{i 0}}
$$

Combining (17), (25) and (26), $K_{\lambda i}, K_{v i}$ can be written as:

$$
\begin{aligned}
K_{\lambda i} & =u_{i 0}^{T} A_{s} v_{i 0}=\frac{\partial \Delta \alpha_{i}}{\partial \Delta P_{G j}}+\mathrm{j} \frac{\partial \Delta \beta_{i}}{\partial \Delta P_{G j}} \\
K_{v i} & =\frac{v_{i 1}}{\Delta P_{G}}
\end{aligned}
$$

where $i \in S_{L}$, and $K_{\lambda i}$ can be described by the 1st-order eigenvalue sensitivity $\partial \Delta \alpha / \partial \Delta P_{G}$ and $\partial \Delta \beta / \partial \Delta P_{G}$.

$v_{i 1}$ is given as:

$$
v_{i 2}=\sum_{j=1}^{S_{l}} \alpha_{j} v_{j 0}
$$

Combining (21) and (29), the 2nd-order eigenvalue can be expressed:

$$
A_{0} \sum_{j=1}^{S_{l}} \alpha_{j}^{*} v_{j 0}+\Delta A v_{i 1}=\lambda_{i 0} \sum_{j=1}^{S_{l}} \alpha_{j}^{*} v_{j 0}+\lambda_{i 2} v_{i 0} v_{i 1}
$$

Equation (30) is multiplied by the left eigenvector $u_{i 0}$ :

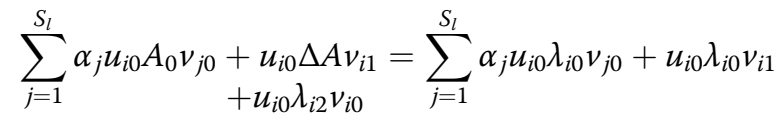

Utilizing orthogonality relation of eigenvector, (31) is derived to:

$$
\lambda_{i 2}=u_{i 0}^{T} \Delta A v_{i 1}-\lambda_{i 1} u_{i 0}^{T} v_{i 1}
$$

Utilizing (17), (27) and (28), (32) can be express:

$$
\begin{aligned}
& \lambda_{i 2}=\Delta P_{G}^{T} u_{i 0}^{T} A_{s} K_{v i} \Delta P_{G}-\Delta P_{G}^{T} K_{\lambda i} u_{i 0}^{T} K_{v i} \Delta P_{G} \\
& =\Delta P_{G}{ }^{T} h_{1} \Delta P_{G}+\Delta P_{G}{ }^{T} h_{2} \Delta P_{G} \\
& h=h_{1}+h_{2}
\end{aligned}
$$

where matrix $h$ is symmetric and the Hessian matrix of eigenvalue $H_{\lambda i}$ are given as:

$$
H_{\lambda i}=h+h^{T}=\frac{\partial^{2} \Delta \alpha_{i}}{\partial \Delta P_{G}{ }^{2}}+j \frac{\partial^{2} \Delta \beta_{i}}{\partial \Delta P_{G}{ }^{2}}
$$

Table 1 The 9-bus system initial operation condition

\begin{tabular}{lccc}
\hline Initial state & Generator 1 & Generator 2 & Generator 3 \\
\hline Active power $(\mathrm{MW})$ & 0.7164 & 1.63 & 0.85 \\
\hline
\end{tabular}

$$
\lambda_{i 2}=\Delta P_{G}^{T} H_{\lambda i} \Delta P_{G}
$$

(4) The gradient matrix and the Hessian matrix of damping ratio

With the 2nd-order Taylor expansion of damping ratio:

$$
\xi_{i}=\xi_{i 0}+K_{\xi i} \Delta P_{G}+\frac{1}{2} \Delta P_{G}^{T} H_{\xi i} \Delta P_{G}
$$

where $\xi_{i 0}$ is the $i$ th initial damping ratio. Damping ratio describes the system stability and is calculated by (18). The first and second-order sensitivities of damping ratio are derived by (27), (28) and (33) considering $i \in S_{L},(k, j) \in S_{G}$ as:

$$
\begin{aligned}
K_{\xi_{i}}= & \frac{\partial \Delta \xi_{i}}{\partial \Delta P_{G j}}=\frac{1}{\sqrt{\alpha_{i}{ }^{2}+\beta_{i}{ }^{2}}} \frac{-\beta_{i}{ }^{2}}{\alpha_{i}{ }^{2}+\beta_{i}{ }^{2}} \frac{\partial \Delta \alpha}{\partial \Delta P_{G j}} \\
& +\frac{1}{\sqrt{\alpha_{i}{ }^{2}+\beta_{i}{ }^{2}}} \frac{\alpha_{i} \beta_{i}}{\alpha_{i}{ }^{2}+\beta_{i}{ }^{2}} \frac{\partial \Delta \beta_{i}}{\partial \Delta P_{G j}}
\end{aligned}
$$

$$
H_{\xi_{i}}=\frac{\partial^{2} \Delta \xi_{i}}{\partial \Delta P_{G j} \partial \Delta P_{G j}}=\frac{\partial^{2} \Delta \xi_{i}}{\partial \Delta P_{G j} \partial \Delta P_{G j}}(1)+\frac{\partial^{2} \Delta \xi_{i}}{\partial \Delta P_{G j} \partial \Delta P_{G j}}(2)
$$

$$
\begin{aligned}
\frac{\partial^{2} \Delta \xi}{\partial \Delta P_{G k} \partial \Delta P_{G j}}(1) & =\frac{-2 \beta_{i}^{*} \frac{\partial \Delta \beta_{i}{ }^{*}}{\partial \Delta P_{G j}} \frac{\partial \Delta \alpha_{i}}{\partial \Delta P_{G k}}-\beta^{2 *} \frac{\partial^{2} \Delta \alpha_{i}}{\partial \Delta P_{G k} \partial \Delta P_{G j}}}{\left(\alpha_{i}{ }^{2}+\beta_{i}{ }^{2}\right)^{3}} \\
& *\left(\alpha_{i}{ }^{2}+\beta_{i}{ }^{2}\right)^{3 / 2}-\frac{-\beta_{i}^{2 *} \frac{\partial \Delta \alpha_{i} * 3}{\partial \Delta P_{G k}} \frac{3}{2}\left(\alpha_{i}{ }^{2}+\beta_{i}{ }^{2}\right)^{1 / 2}}{\left(\alpha_{i}{ }^{2}+\beta_{i}\right)^{3}} \\
& * \frac{\left(2 \alpha_{i} \frac{\partial \Delta \alpha_{i}}{\partial \Delta P_{G j}}+2 \beta_{i} \frac{\partial \Delta \beta_{i}}{\partial \Delta P_{G j}}\right)}{\left(\alpha_{i}{ }^{2}+\beta_{i}{ }^{2}\right)^{3}}
\end{aligned}
$$

Table 2 The 9-bus system unstable state

\begin{tabular}{llcl}
\hline System state & Eigenvalue & Damping ratio & Oscillation frequency \\
\hline Modal 1 & $-0.1515 \pm j 8.6712$ & 0.0175 & $1.380057328 \mathrm{~Hz}$ \\
\hline
\end{tabular}


Table 3 Adjustment results based MPT ( $\left.\xi_{\mathrm{r}}=0.032\right)$

\begin{tabular}{llll}
\hline Sensitivity & Eigenvalue & $\begin{array}{l}\text { Oscillation } \\
\text { frequency }\end{array}$ & $\begin{array}{l}\text { Damping } \\
\text { ratio }\end{array}$ \\
\hline $\begin{array}{l}\text { 1st-order } \\
\text { sensitivity }\end{array}$ & $-0.320 \pm j 9.199$ & $1.4641 \mathrm{~Hz}$ & 0.0348 \\
$\begin{array}{l}\text { 2nd-order } \\
\text { sensitivity }\end{array}$ & $-0.304 \pm j 9.201$ & $1.4644 \mathrm{~Hz}$ & 0.0330 \\
\hline
\end{tabular}

$$
\begin{aligned}
& \frac{\partial^{2} \Delta \xi_{i}}{\partial \Delta P_{G k} \partial \Delta P_{G j}}(2)=\frac{\frac{\partial \Delta \alpha_{i}}{\partial \Delta P_{G j}} \beta_{i}^{*} \frac{\partial \Delta \alpha_{i}}{\partial \Delta P_{G k}}+\alpha^{*} \frac{\partial \Delta \beta_{i}{ }^{*}}{\partial \Delta P_{G j}} \frac{\partial \Delta \beta_{i i}}{\partial \Delta P_{G k}}}{\left(\alpha_{i}{ }^{2}+\beta_{i}{ }^{2}\right)^{3 / 2}} \\
& +\frac{\alpha_{i} \beta_{i} \frac{\partial_{i}^{2} \Delta \beta_{i}}{\partial \Delta P_{G k} \partial \Delta P_{G j}}}{\left(\alpha_{i}{ }^{2}+\beta_{i}{ }^{2}\right)^{3 / 2}}-\frac{\alpha_{i} \beta_{i} \frac{\partial \Delta \beta_{i}}{\partial \Delta P_{G k}} \frac{3}{2}\left(\alpha_{i}{ }^{2}+\beta_{i}^{2}\right)^{1 / 2}}{\left(\alpha_{i}{ }^{2}+\beta_{i}{ }^{2}\right)^{3}} \\
& * \frac{\left(2 \alpha_{i} \frac{\partial \Delta \alpha_{i}}{\partial \Delta P_{G j}}+2 \beta_{i} \frac{\partial \Delta \beta_{i}}{\partial \Delta P_{G j}}\right)}{\left(\alpha_{i}^{2}+\beta_{i}^{2}\right)^{3}}
\end{aligned}
$$

To utilize (39), the expression of the Hessian matrix $H_{\xi i}$ is:

$$
H_{\xi_{i}}=\left[\begin{array}{ccc}
\frac{\partial^{2} \xi_{i}}{\partial P_{G 1}^{2}} & \cdots & \frac{\partial^{2} \xi_{i}}{\partial P_{G j} \partial P_{G 1}} \\
\ddot{\partial}^{2} \ddot{\xi}_{i} & \cdots & \ddot{\partial}^{2} \ddot{\xi}_{i} \\
\frac{\partial P_{G 1} \partial P_{G j}}{\partial P_{G j}^{2}} & \cdots &
\end{array}\right]
$$

The $H_{\xi i}$ and $K_{\xi i}$ are deduced by the sensitivity of state matrix $A_{s}$ and the initial eigenfunction. The sensitivity of state matrix $A_{s}$ is deduced by the change of state matrix $\Delta A$ and perturbation variable $\Delta P_{G}$. Because system stability is estimated by the state matrix $A_{0}$, the initial eigenfunction is known.

The existing approach [10] contains heavy eigenvalue computation used in the QR method, and thus, the computation speed of QR method increases with the size of state matrix. The QR algorithm is an eigenvalue algorithm: that is, a procedure to calculate the eigenvalues and eigenvectors of a matrix. The basic idea is to perform QR decomposition, writing the matrix as a product of an orthogonal matrix and an upper triangular matrix, multiply the factors in the reverse order, and iterate. The sensitivity matrices $A_{s}$ are obtained through the perturbation variable and the change of state matrix $\Delta A$ in the proposed method. The computational process of $H_{\xi i}$ and $K_{\xi i}$ only contains simple multiplications and divisions, and thus simplifies the calculation procedure. Compared

Table 4 Test results of NES-SSSC-OPF and MPT-OS

\begin{tabular}{lll}
\hline Method & Minimum damping ratio & Computation time \\
\hline NES-SSSC-OPF & 0.0347 & $0.0233 \mathrm{~s}$ \\
MPT-OS & 0.0330 & $0.0182 \mathrm{~s}$ \\
\hline
\end{tabular}

Table 5 Generator actual active adjustment

\begin{tabular}{lllll}
\hline $\begin{array}{l}\text { Damping } \\
\text { threshold }\end{array}$ & $\begin{array}{l}\text { 1st-Oder } \\
\text { power } \\
\text { adjustment }\end{array}$ & $\begin{array}{l}\text { 2nd-Oder } \\
\text { power } \\
\text { adjustment }\end{array}$ & $\begin{array}{l}\text { 1st-Oder } \\
\text { minimum } \\
\text { damping ratio }\end{array}$ & $\begin{array}{l}\text { 2nd-Oder } \\
\text { minimum } \\
\text { damping ratio }\end{array}$ \\
\hline 0.03 & $0.49 \mathrm{MW}$ & $0.444 \mathrm{MW}$ & 0.034 & 0.0330 \\
\hline
\end{tabular}

with the existing methods $[12,13,15]$, burdensome deviation calculations of damping ratio are avoided in the presented method, and the calculation steps are direct and explicit. Thus, the method has higher efficiency for high order state matrix calculation.

In order to obtain the eigenvalue sensitivities in (17), (25), (32), $\Delta A$ needs to be calculated by $A_{0}$ and $A$, while system voltage obtained by active power perturbation can constitute $A$ by (13) in perturbation process. In process of active power perturbation, node voltage is obtained by time-consuming power flow calculations, whereas the system voltage is calculated by power sensitivity so as to avoid repeated power flow calculations.

\subsection{The power sensitivity application}

The system voltage can describe the system state and the state matrix is established by the system state. The voltage can be calculated by power sensitivity so that the iteration time is saved in power flow calculation.

$J a$ is the Jacobian matrix in the Newton-Raphson method $[29,30]$ and the derivatives of voltage can be derived by the Jacobian matrix $\mathrm{Ja}$ from (5). The derivatives of the voltage amplitude and phase angle can be written as:

$$
\begin{aligned}
& {\left[\begin{array}{l}
\frac{\partial \Delta V}{\partial \Delta P} \\
\frac{\partial \Delta \theta}{\partial \Delta P}
\end{array}\right]=J a^{-1} \frac{\partial \Delta S}{\partial \Delta P}} \\
& V^{\prime}=V+\frac{\partial \Delta V}{\partial \Delta P} \Delta P \\
& \theta^{\prime}=\theta+\frac{\partial \Delta \theta}{\partial \Delta P} \Delta P
\end{aligned}
$$

where $\partial \Delta V / \partial \Delta P$ and $\partial \Delta \theta / \partial \Delta P$ are the voltage amplitude sensitivity and phase angle sensitivity, respectively. $\Delta \theta$ and $\Delta V$ are the changes of system voltage amplitude and phase angle, respectively. $\Delta S$ and $\Delta P$ are the respective power changes in the system and generator, and $\partial \Delta S /$ $\partial \Delta P=[0, \ldots .0,1,0, \ldots 0]^{T}$.

Utilizing the voltage sensitivities in (44) and (45), new state matrix $A$ can be obtained by (13). Then, $\Delta A$

Table 6 The 39-bus system unstable state

\begin{tabular}{llll}
\hline State & Eigenvalue & Damping ratio & Frequency $(\mathrm{Hz})$ \\
\hline Modal 1 & $-0.1932 \pm \mathrm{j} 6.8722$ & 0.0281 & 1.0938 \\
Modal 2 & $-0.1742+\mathrm{j} 6.7736$ & 0.0257 & 1.0738 \\
Modal 3 & $-0.0143 \pm \mathrm{j} 0.9386$ & 0.0153 & 0.1494 \\
\hline
\end{tabular}




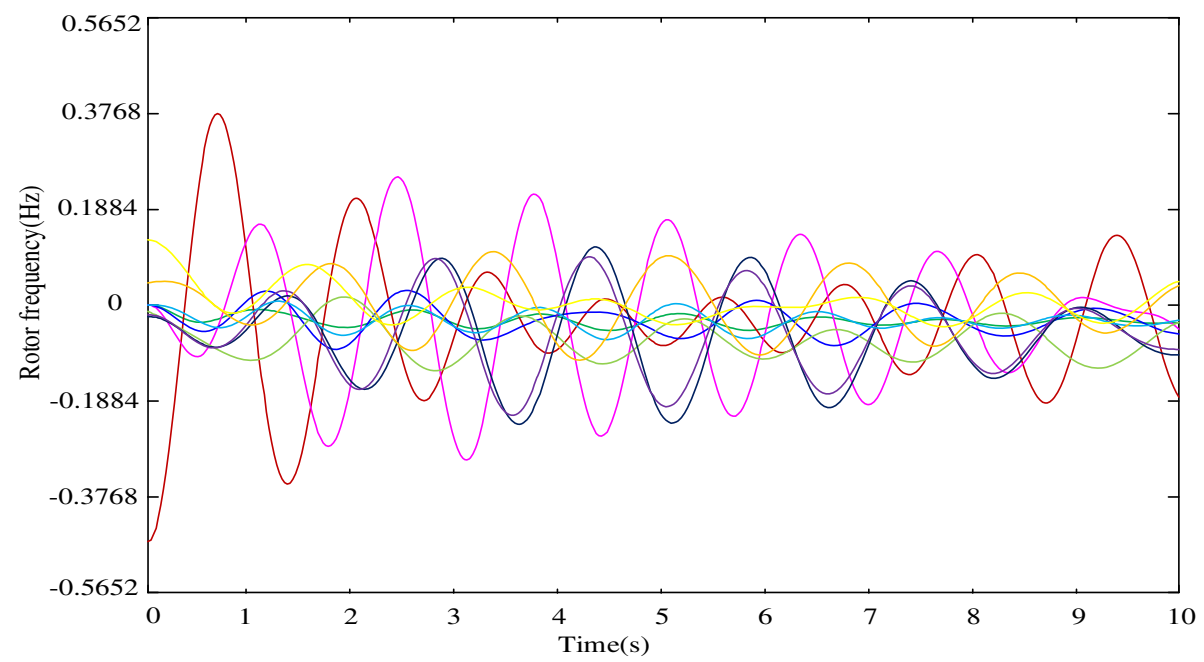

Fig. 2 Rotor frequency oscillation deviations. The Figure shows the deviations of rotor frequency oscillation in initial system, after the system is disturbed by disturbance of $10 \%$ power load

required in the above section can be calculated by $A_{0}$ and $A$ in (16). The method avoids iterative computation of power flow, which greatly reduce required calculation.

\section{Results and discussion}

Following examples are built on the MATLAB platform. The WSCC 3-machine 9-bus, New England 10-machine 39-bus and the China 124-machine 634-bus systems are used for illustrating the proposed technique. The convergence precision is $10^{-4}$ and damping ratio limit $\xi$ is 0.03 .

\subsection{The 9-bus 3-machine system}

In modeling the system, the generator model is a 6th-order model, the excitation model is a self-shunt

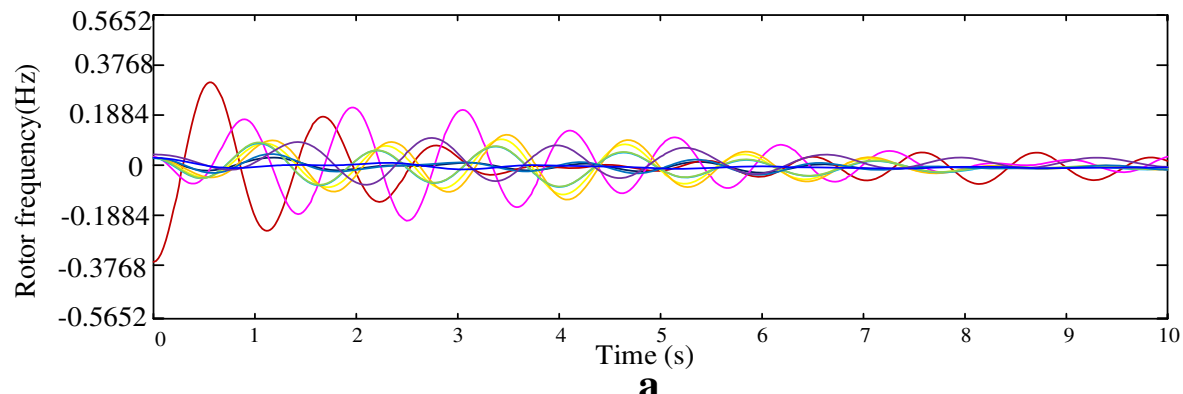

a

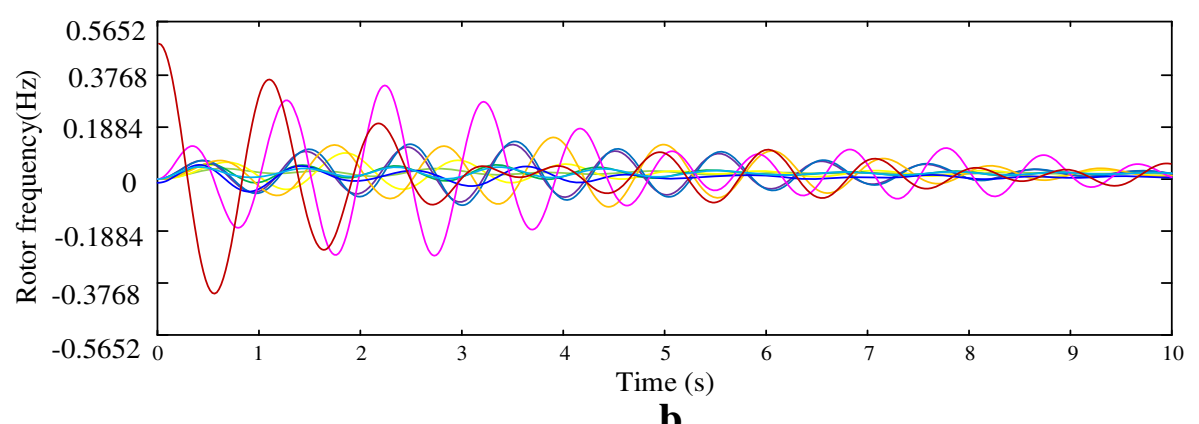

b

Fig. 3 Rotor frequency deviations after improving system stability: a 1-st order sensitivity MPT, b 2nd-order sensitivity MPT. The Figure show that rotor frequency deviations after the system is disturbed by disturbance of 10\% power load. System stability has been improved by two kinds of re-dispatch 
Table 7 Adjustment results based MPT (1st-order sensitivity)

\begin{tabular}{llll}
\hline State & Eigenvalue & Damping ratio & Frequency $(\mathrm{Hz})$ \\
\hline Modal 1 & $-0.1946 \pm j 6.0854$ & 0.032 & 1.0322 \\
Modal 2 & $-0.1794 \pm j 5.3150$ & 0.033 & 1.18156 \\
Modal 3 & $-0.1972 \pm j 5.6199$ & 0.0351 & 0.8944 \\
\hline
\end{tabular}

static model and the load is modelled as constant resistors. Through the small-signal stability analysis, Tables 1 and 2 show the initial operation conditions.

The oscillation frequencies of multiple modes are in the range of $0-2.4 \mathrm{~Hz}$ in the original system with only one damping ratio state being less than 0.03 at 0.0175 . Thus, the system is prone to low frequency oscillation after disturbance.

The above system stability is improved by the proposed methodology. Tables 3, 4, and 5 show the optimal results $(\xi=0.03)$. The index $\xi_{\text {err }}$ is to evaluate the advantages of the 2nd-order damping, and is defined as the relative error of damping as:

$$
\xi_{\mathrm{err}}=\left|\xi_{t}-\xi_{r}\right| / \xi_{t}
$$

where $\xi_{\mathrm{t}}$ is the minimum true value of damping after system calibration, and $\xi_{\mathrm{r}}$ is the result of correction strategy.

As can be seen from Table 3, the minimum damping ratio is 0.033 , which is greater than $\xi_{\text {limit }}$ of 0.03 . The system can suppress oscillation in a short time after stability correction. For (46), the relative error is 3.125\% and the numerical error is 0.001 in minimum damping ratio, when considering the Hessian matrix of damping ratio in the model. However, the corresponding relative error is $6.25 \%$ and the numerical error is 0.002 when considering the gradient matrix of damping ratio. Table 4 shows the test results of NES-SSSC-OPF and MPT-OS, indicating that MPT-OS is faster than NES-SSSC-OPF in computation time.

Table 5 shows the relationship between the damping ratio and the active power adjustment. The increase in damping ratio will increase the generator active output adjustment. The active power adjustment of the 1st-order sensitivity is higher than that of the 2nd-order sensitivity. This is due to the control strategy only considering the damping ratio gradient matrix, which has lower accuracy than the Hessian matrix. Similarly, the

Table 8 Adjustment results based MPT (2nd-order sensitivity)

\begin{tabular}{llll}
\hline State & Eigenvalue & Damping ratio & Frequency $(\mathrm{Hz})$ \\
\hline Modal 1 & $-0.1594 \pm j 5.3150$ & 0.03 & $0.8459 \mathrm{~Hz}$ \\
Modal 2 & $-0.1954 \pm j 5.8811$ & 0.0332 & $0.936 \mathrm{~Hz}$ \\
Modal 3 & $-0.2772 \pm j 8.1952$ & 0.0338 & $1.2052 \mathrm{~Hz}$ \\
\hline
\end{tabular}

Table 9 Test Results of NES-SSSC-OPF and MPT-OS ( $\left.\xi_{\mathrm{r}}=0.030\right)$

\begin{tabular}{lll}
\hline Method & Minimum damping ratio & Computation time \\
\hline NES-SSSC-OPF & 0.033 & $0.2324 \mathrm{~s}$ \\
MPT-OS & 0.030 & $0.1481 \mathrm{~s}$
\end{tabular}

1st-order relative error is always bigger than that of the 2nd-order in minimum damping ratio.

\subsection{The 39-bus 10-machine system}

In the system model, the generators also adopt the 6th-order model, the excitation system is a fast-static excitation model and the constant resistance model is also used.

From small-signal stability analysis, the damping ratios below 0.03 contain $0.0281,0.0257$ and 0.0153 as shown in Table 6.

The system may become unstable due to insufficient damping. Generators have the ability to provide additional measure to the system to maintain the small-signal stability. The 39-bus system is adjusted by NES-SSSC-OPF and MPT-OS respectively, and Tables 7, 8, 9, 10 and 11 describes the adjustment results.

The 1 st-order $\xi_{\text {err }}$ is $6.7 \%$ and the 2 nd-order $\xi_{\text {err }}$ is $0 \%$ according to (46), and active power adjustment of the 2nd-order sensitivity is smaller than that of the 1st-order one. Comparing the two methods, the NES-SSSC-OPF is more time consuming than the MPT-OS shown in Table 9. The eigenfunction problem is solved by QR algorithm with significant computation.

In Fig. 2, the rotor frequencies fluctuate severely at $10 \mathrm{~s}$, and its maximum frequency deviation is $0.1884 \mathrm{~Hz}$. This is due to the small damping ratio of the initial system and thus the system cannot quickly recover to the initial state. By contrast, Fig. 3 shows the rotor frequency deviations after a $10 \%$ load disturbance. System

Table 10 Adjustment of active power (1st-order sensitivity)

\begin{tabular}{llll}
\hline Number & Initial state & The proposed strategy & Adjustment \\
\hline G1(MW) & 2.5 & 2.9729 & 0.4729 \\
G2(MW) & 5.7323 & 2.1407 & 3.5916 \\
G3(MW) & 6.5 & 2 & 4.5 \\
G4(MW) & 6.32 & 8.0377 & 1.7177 \\
G5(MW) & 5.08 & 7.4301 & 2.3501 \\
G6(MW) & 6.5 & 7.4301 & 0.9301 \\
G7(MW) & 5.6 & 7.3428 & 1.7428 \\
G8(MW) & 5.4 & 5.8269 & 0.4269 \\
G9(MW) & 8.3 & 8.9056 & 0.6056 \\
G10(MW) & 10 & 11.3054 & 1.3054 \\
Total (MW) & 61.9323 & 63.3922 & 17.6431 \\
\hline
\end{tabular}


Table 11 Adjustment of active power (2nd-order sensitivity)

\begin{tabular}{llll}
\hline Number & Initial state & The proposed strategy & Adjustment \\
\hline G1(MW) & 2.5 & 3.1166 & 0.6166 \\
G2(MW) & 5.7323 & 7.6808 & 1.9485 \\
G3(MW) & 6.5 & 2.1827 & 4.3173 \\
G4(MW) & 6.32 & 7.0752 & 0.7552 \\
G5(MW) & 5.08 & 5.691 & 0.611 \\
G6(MW) & 6.5 & 6.978 & 0.478 \\
G7(MW) & 5.6 & 6.5624 & 0.9624 \\
G8(MW) & 5.4 & 6.2396 & 0.8396 \\
G9(MW) & 8.3 & 5.696 & 2.604 \\
G10(MW) & 10 & 11.1497 & 1.1497 \\
Total (MW) & 61.9323 & 62.372 & 14.2823 \\
\hline
\end{tabular}

stability has been improved by the two re-dispatch plans, one has the 1st-order damping sensitivity and the other 2nd-order damping sensitivity. At 20s, rotor frequency deviation with the 1st-order damping sensitivity is smaller than that with the 2nd-order damping sensitivity. Compared with Fig. 2, the fluctuation of rotor frequency is smaller in Fig. 3. The maximum frequency deviation is $0.0328 \mathrm{~Hz}$ in Fig. 3a compared to $0.0358 \mathrm{~Hz}$ in Fig. 3b. The damping ratio of the 1st-order sensitivity is higher than that of the 2nd-order sensitivity, resulting in reduced rotor frequency deviation of the 1st-order sensitivity compared to that of the 2nd-order sensitivity.

From Tables 7, 8, 10 and 11, the active power adjustment of generators and damping ratio variable $\Delta \xi$ gradually decline with the increase of the order of sensitivity. The main reason for this is that high order sensitivity allows more accurate control of damping ratio.

From Tables 10 and 11, it is seen that the active power adjustment results satisfy the small-signal stability constraints by MPT-OS. In addition, the adjustment results with the 2nd-order sensitivity is close to actual adjustment results in the control model, while damping ratio of the 1st-order sensitivity is higher than that of the 2nd-order sensitivity. This means that, the system can provide more damping ratio, its stability is superior to

Table 12 634-bus system operation condition

\begin{tabular}{llll}
\hline State & Eigenvalue & Damping & Frequency \\
\hline Modal 1 & $-0.6028+j 14.5467$ & 0.0414 & $2.31513608 \mathrm{~Hz}$ \\
Modal 2 & $-0.5486+j 14.4215$ & 0.0381 & $2.295081967 \mathrm{~Hz}$ \\
Modal 3 & $-0.5134+\mathrm{j} 13.6761$ & 0.0375 & $2.176667197 \mathrm{~Hz}$ \\
Modal 4 & $2.1815+\mathrm{j} 0.0000$ & -1 & $0 \mathrm{~Hz}$ \\
Modal 5 & $12.6029+\mathrm{j} 0.0000 \mathrm{i}$ & -1 & $0 \mathrm{~Hz}$ \\
$\ldots . .$. & $\ldots \ldots .$. & $\ldots \ldots$. & $\ldots \ldots$. \\
\hline
\end{tabular}

Table 13 Test results of Chinese 634-bus system

\begin{tabular}{lll}
\hline Method & $\begin{array}{l}\text { Min damping } \\
\text { ratio }\end{array}$ & Computation time \\
\hline NES-SSSC-OPF & 0.0372 & $61.5861 \mathrm{~s}$ \\
MPT-OS & 0.0350 & $21.2479 \mathrm{~s}$ \\
\hline
\end{tabular}

the original state, and it can suppress rotor frequency fluctuation in a shorter time.

\subsection{The 634-bus 124-machine system}

The actual power grid in China is taken as an example, which has 634 nodes, 532 lines, 124 generators, 190 reactive power compensation points and 879 transformers. In the modelled system, the generators use the 6th-order model, the load is again modelled as constant load and the excitation model is the fast-static excitation.

The initial operating condition in Table 12 shows unstable modes with damping ratio less than the threshold of 0.03. Thus, if the system is subject to small disturbance, system is prone to oscillation.

With the optimal model in Section 2, the stability of the system is improved by the output power of generating units.

Comparing Tables 4, 9 and 13, it can be seen that MPT-OS is faster than NES-SSSC-OPF in computation time while increasing the system scale leads to increased difference of the total CPU time between the two. It can be concluded that MPT-OS has higher efficiency for high order state matrix calculation than NES-SSSC-OPF.

After system damping ratio improvement, $\xi_{\mathrm{r}}$ is 0.034 . As can be seen from Table 14, the minimum damping ratio is 0.0372 when the optimal strategy considers the 1st-order sensitivity. However, the minimum damping ratio is 0.0350 when the 2 nd-order sensitivity is considered. From (46), $\xi_{\text {err }}$ is $8.6 \%$ with the 1 st-order sensitivity and is $2.8 \%$ with the 2 nd-order sensitivity. The active power adjustment with 2nd-order sensitivity is smaller than that with the 1st-order sensitivity. This is because high order sensitivity can allow a more accurate control of damping ratio. It can be seen from the above table that, compared to the 1st-order sensitivity, the 2nd-order sensitivity more accurately characterizes damping ratio state, leading to better precision.

Table 14 Adjustment results based MPT $\left(\xi_{\mathrm{r}}=0.034\right)$

\begin{tabular}{llll}
\hline Sensitivity & Eigenvalue & $\begin{array}{l}\text { Minimum } \\
\text { damping ratio }\end{array}$ & $\begin{array}{l}\text { Active } \\
\text { adjustment }\end{array}$ \\
\hline 1st-order & $-0.5400+j 14.4385$ & 0.0372 & $0.5173 \mathrm{MW}$ \\
2nd-order & $-0.0069+\mathrm{j} 0.1337$ & 0.0350 & $0.5064 \mathrm{MW}$ \\
\hline
\end{tabular}




\section{Conclusion}

This paper has showed that optimization strategy with constraints for small-signal stability can improve system stability by using methods from Matrix Perturbation Theory (MPT). First, a small signal analysis is used to identify the degree of influence of unstable modes on the system. The desired sensitivity matrix is then calculated through MPT, which can describe damping ratio constraints. In the optimized corrective control, a series of comprehensive restrictions are proposed to enhance the system damping ratio while meeting the normal operation requirements.

The strategy presented in this paper does not require burdensome deviation calculation to describe the 2nd-order damping ratio sensitivity. The desired sensitivity can be obtained by perturbation variable and the change of the state matrix. The solving steps are direct and explicit, and the calculation of high order sensitivity matrix is simplified. Furthermore, the active power adjustment with the 1st-order sensitivity is higher than that with the 2nd-order sensitivity in the optimization strategy. Since the accuracy of the gradient matrix is lower than that of the Hessian matrix, high order sensitivity can gain higher accuracy in controlling damping ratio and active power adjustment. Compared to other methods, the proposed MPT based optimal strategy can avoid the deviation calculation and reduce computation time, while ensuring calculation accuracy.

\section{Abbreviations}

IPM: Interior Point Method; MPT: Matrix Perturbation Theory; SSC-OPF: SmallSignal-Stability Constrained Optimal Power Flow

\section{Funding}

This work was supported by the National Natural Science Foundation of China (Grant No.51577085).

\section{Authors' contributions}

YY conceived and designed the study. YY and JZ performed the experiments and simulations. YY, JZ and HL wrote the paper. YY, JZ, HL, ZQ, JD and JQ reviewed and edited the manuscript. All authors read and approve the manuscript.

\section{Competing interests}

The authors declare that they have no competing interests.

\section{Author details}

${ }^{1}$ College of Electrical Engineering, Guangxi University, Nanning 530004, China. ${ }^{2}$ State Grid Shaanxi Electric Power Research Institute, Xi'an, China. ${ }^{3}$ Department of Electrical and Computer Engineering, University of Central Florida, Orlando, FL 32816, USA.

Received: 8 March 2018 Accepted: 26 September 2018 Published online: 02 November 2018

\section{References}

1. Zhu, F., Hongguang, Z., Zenghuang, L., et al. (2007). The influence of large power grid interconnected on power system dynamic stability. Proceedings of the CSEE, 27(1), 1-7.
2. Yu, Y., \& Peng, L. (2005). The impact of weak interconnection of bulk power grids to damping and dynamic stability of power systems. Proceedings of the CSEE, 25(11), 6-11.

3. Yu, Z., Fang, L., Lu, S., et al. (2014). The impact of weak internection of bulk power grids to damping and dynamic stability of power systems. Proceedings of the CSEE, 34(34), 6191-6198.

4. Lihong, G., Xiaoxin, Z., Jianfeng, Y., et al. (2010). Real time small signal stability analysis of power systems interconnected. Proceedings of the CSEE, 30(13), 1-7.

5. Wang, C., et al. (2015). Low frequency oscillation characteristics of East China power grid after commissioning of Huai-Hu ultra-high voltage alternating current project. Journal of Modern Power Systems and Clean Energy, 3(3), 332-340.

6. Quintero, J., Vittal, V., Heydt, G., \& Zhang, H. (2014). The impact of increased penetration of converter control-based generators on power system modes of oscillation. IEEE Transactions on Power Apparatus and Systems, 29(5), 2248-2256.

7. Wei, S., Zhou, Y., \& Huang, Y. (2017). Synchronous motor-generator pair to enhance small signal and transient stability of power system with high penetration of renewable energy. IEEE Access, 5, 11505-11512.

8. Su, C., Hu, W., Chen, Z., \& Hu, Y. (2013). Mitigation of power system oscillation caused by wind power fluctuation. IET Renewable Power Generation, 7(6), 639-651.

9. Lee, D. J., \& Wang, L. (2008). Small-signal stability analysis of an autonomous hybrid renewable energy power generation/energy storage system part I: time-domain simulations. IEEE Transactions on Energy Conversion, 23(1), 311-320.

10. Chung, C. Y., Wang, L., Howell, F., \& Kundur, P. (2004). Generation rescheduling methods to improve power transfer capability constrained by small-signal stability. IEEE Transactions on Power Apparatus and Systems, 19(1), 524-530.

11. Zarate-Minano, R., Milano, F., \& Conejo, A. J. (2011). An OPF methodology to ensure small-signal stability. IEEE Transactions on Power Apparatus and Systems, 26(3), 1050-1061.

12. Condren, J., \& Gedra, T. (2006). Expected-security-cost optimal power flowwith small-signal stability constraints. IEEE Transactions on Power Apparatus and Systems, 21(4), 1736-1743.

13. Kodsi, S., \& Caizares, C. (2007). Application of a stability-constrained optimal power flow to tuning of oscillation controls in competitive electricity markets. IEEE Transactions on Power Apparatus and Systems, 22(4), 1944-1954.

14. Curtis, F. E., \& Overton, M. L. (2012). A sequential quadratic programming algorithm for nonconvex, nonsmooth constrained optimization. SIAM Journal on Optimization, 22(2), 474-500.

15. Li, P., Qi, J., Wang, J., Wei, H., Bai, X., \& Qiu, F. (2017). An SQP method combined with gradient sampling for small-signal stability constrained OPF. IEEE Transactions on Power Apparatus and Systems, 32(3), 2372-2381.

16. Gunel, S., \& Zoral, E. Y. (2010). Parametric history analysis of resonance problems via step-by-step eigenvalue perturbation technique. IET Microwaves Antennas and Propagation, 4(4), 466-476.

17. Ma, J., Peng, M., Wang, T., \& Yang, Q. (2007). Small signal stability analysis based on second order perturbation theory. Electric Power Automation Equipment, 33(7), 117-125.

18. Liu, H., Zhao, W., Zhang, J., Wang, L., Ma, X., \& Zhao, F. (2011). Modal analysis of machine tools during working process by matrix perturbation method, 2011 IEEE International Symposium on Assembly and Manufacturing (ISAM) (pp. 1-4).

19. Chen, S. H. (2007). Matrix Perturbation Theory in Structural Dynamic Design. Beijing: Science

20. G. S. Happawan, A. K. Bajaj, and O. D. I. Nwokah. (1993). Modal analysis and forced response of coupled mistuned cyclic systems: A singular perturbation approach. Proc. Int. Gas Turbine Aeroengine Congr., Cincinnati, OH, USA, V03BT16A022-1-V03BT16A022-13.

21. Wang, C., Li, Y., Peng, K., Hong, B., Wu, Z., \& Sun, C. (2013). Coordinated optimal design of inverter controllers in a micro-grid with multiple distributed generation units. IEEE Transactions on Power Apparatus and Systems, 28(3), 2679-2687.

22. Chatterjee, A. (2016). Sensitivity and error analysis of a coupled micro-resonator array for ultra-sensitive mass detection using matrix perturbation theory. IEEE Sensors Journal, 16(5), 1304-1309.

23. Chatterjee, A., \& Kotambkar, M. S. (2015). Modal characteristics of turbine blade packets under lacing wire damage induced mistuning. Journal of Sound and Vibration, 343, 49-70. 
24. Tong, W., Ma, J., Zengping, W., et al. (2013). Multiple parameter modal analysis of power system low-frequency oscillation based on the 2nd order sensitivity matrix. Proceedings of the CSEE, 33(34), 205-213.

25. Assem, S. D. (1982). Advanced matrix theory for scientists and engineers. Kent: Abacus.

26. Tao, L., Xinli, S., Yong, T., et al. (2000). A new eigenvalue sensitivity theory of augmented matrix and its applications to power system stability analysis. IEEE Transactions on Power Systems, 15(5), 363-369.

27. Wang, Y., Lu, Z. X., Min, Y., \& Wang, Z. (2011). Small signal analysis of microgrid with multiple micro sources based on reduced order model in islanding operation. In IEEE Power Energy Society General Meeting (pp. 1-9).

28. Chung, I.-Y., Liu, W. X., Cartes, D. A., Collins, E. G., \& Moon, S.-I. (2010). Control methods of inverter-interfaced distributed generators in a microgrid system. IEEE Transactions on Industry Applications, 46(3), 1078-1088.

29. Zhang, Z., Ochoa, L. F., \& Valverde, G. (2018). A novel voltage sensitivity approach for the decentralized control of DG plants. IEEE Transactions on Power Apparatus and Systems, 33(2), 1566-1576.

30. Konar, J. S., Chatterjee, D., \& Patra, S. (2015). V-Q sensitivity-based index for assessment of dynamic voltage stability of power systems. IET Generation Transmission and Distribution, 9(7), 677-685.

\section{Submit your manuscript to a SpringerOpen ${ }^{\mathcal{O}}$ journal and benefit from:}

- Convenient online submission

- Rigorous peer review

- Open access: articles freely available online

- High visibility within the field

Retaining the copyright to your article

Submit your next manuscript at $\boldsymbol{\nabla}$ springeropen.com 